\title{
Mesonephric-Like Adenocarcinoma of the Endometrium: Diagnostic Advances to Spot This Wolf in Sheep's Clothing. A Review of the Literature
}

\author{
Ellen Deolet ${ }^{1}$, Jo Van Dorpe ${ }^{1,2}$ and Koen Van de Vijver $1,2, *$ (D) \\ 1 Department of Pathology, Ghent University Hospital, 9000 Ghent, Belgium; ellen.deolet@uzgent.be (E.D.); \\ jo.vandorpe@uzgent.be (J.V.D.) \\ 2 Cancer Research Institute Ghent (CRIG), Ghent University, 9000 Ghent, Belgium \\ * Correspondence: koen.vandevijver@uzgent.be
}

Citation: Deolet, E.; Van Dorpe, J.; Van de Vijver, K. Mesonephric-Like Adenocarcinoma of the Endometrium: Diagnostic Advances to Spot This Wolf in Sheep's Clothing. A Review of the Literature. J. Clin. Med. 2021, 10, 698.

https://doi.org/10.3390/jcm10040698

Academic Editor: Andrea Romano

Received: 31 December 2020

Accepted: 1 February 2021

Published: 11 February 2021

Publisher's Note: MDPI stays neutral with regard to jurisdictional claims in published maps and institutional affiliations.

Copyright: (c) 2021 by the authors. Licensee MDPI, Basel, Switzerland. This article is an open access article distributed under the terms and conditions of the Creative Commons Attribution (CC BY) license (https:// creativecommons.org/licenses/by/ $4.0 /)$.

\begin{abstract}
Mesonephric-like adenocarcinoma is a recently described rare neoplasm occurring in the uterine corpus and ovary. This under-recognized subtype of carcinoma can be very challenging to diagnose. In mesonephric adenocarcinoma a variety of growth patterns can be present within the same tumor, as a result of which they can be misinterpreted and diagnosed as low-grade endometrioid adenocarcinoma, clear cell carcinoma, or even serous carcinoma and carcinosarcoma. We report a case of mesonephric-like adenocarcinoma misdiagnosed as a low-grade endometrioid endometrial adenocarcinoma that had an early local recurrence and metastasized to the liver and the lungs. Histopathological, immunohistochemical and molecular analysis were performed and compared to published literature, providing a comprehensive overview of the current knowledge. Databases (Pubmed, Web of Science, Google Scholar) were searched with a combination of the following search terms: mesonephric-like, mesonephric, adenocarcinoma, carcinoma, uterine body, uterine corpus, endometrium. Mesonephric-like adenocarcinoma is a difficult-to-diagnose entity. Advanced diagnostics, including improved morphologic, immunohistochemical and molecular knowledge can help develop new therapeutic strategies against this specific subtype of endometrial cancer with an aggressive clinical behavior.
\end{abstract}

Keywords: mesonephric-like adenocarcinoma; uterus; endometrium; histology; immunohistochemistry; molecular; KRAS

\section{Introduction}

Mesonephric-like adenocarcinomas (MLAs) represent recently described rare tumors occurring in the uterus and the ovaria. It is still a matter of controversy whether these tumors are of mesonephric origin or represent Müllerian neoplasms closely mimicking mesonephric adenocarcinomas. They show morphological, immunohistochemical and molecular similarities to mesonephric adenocarcinomas (MA) that originate from true mesonephric remnants. They have, however, overlapping features with Müllerian type carcinomas as an association with mesonephric remnants or hyperplasia is not always found and they arise in the endometrium. On a molecular basis, MA and MLA share KRAS mutations, but in MLA, concurrent PIK3CA mutations are described in nearly half of the cases [1], a mutation not found in MA and present among the genetic alterations in endometrioid adenocarcinoma [2]. There are a handful of cases where MLA is associated with other Müllerian neoplasms and clonality between the two is proven as they share identical KRAS or NRAS mutations [3,4]. Whole proteomic analysis, however, could not distinguish MA from MLA [5]. Most importantly, MLA is often misdiagnosed as other endometrial neoplasms, but has an aggressive clinical behavior and tend to metastasize early to the lungs [6-10]. 


\section{Materials and Methods}

We report a case of mesonephric-like adenocarcinoma misdiagnosed as a low-grade endometrioid endometrial adenocarcinoma that had an early local recurrence and metastasized to the liver and the lungs. Histopathological, immunohistochemical and molecular analyses were performed and compared to published literature, providing a comprehensive overview of the current knowledge. Databases (Pubmed, Web of Science, Google Scholar) were searched with a combination of the following search terms: mesonephric-like, mesonephric, adenocarcinoma, carcinoma, uterine body, uterine corpus, endometrium. After reading the title and the abstract, articles in English, where the full text was available, were included. Articles about all mesonephric-like adenocarcinomas (uterine body and ovarian) were included to give an overview of all the current knowledge. The search was performed by one of the authors (ED).

\section{Results}

\subsection{Case Presentation}

A 76-year-old woman was referred to our hospital because of metastasized carcinoma. Her previous clinical history included a rectal adenocarcinoma 22 years ago (1998 TNM '98 pT3N1; treated with rectum resection and adjuvant chemotherapy with 5-FU and Elvorin). Three years ago she was diagnosed in another hospital with a low-grade (grade 1-2) endometrioid endometrial carcinoma with invasion to the inner half of the myometrium, TNM (UICC, 8th edition) pT1a, International Federation of Gynecology and Obstetrics (FIGO, 2009) stage IA. She underwent a total abdominal hysterectomy and bilateral salpingo-oophorectomy. She received adjuvant external radiation therapy of $46 \mathrm{~Gy}$ at the operation field, in the upper third of the vagina and elective lymph-node regions. Five months after primary surgery, she had a local recurrence at the vaginal vault, located in the irradiation field. This local recurrence was treated with simultaneous integrated boost radiation therapy (externally and brachytherapy) up to 62 Gy. Subsequently, 9 months after primary surgery, she developed liver metastasis, histologically consistent with the endometrial adenocarcinoma. The next metastases presented 4 months later, 1 year after primary surgery, both to the liver and the lung. This time she was not only treated with metastasectomy but also with carboplatin-taxol chemotherapy. Now, three years after primary surgery, she presents with a third metastasis, again to the lung.

All metastases showed similar morphology. They were composed of tubules and glandular structures with well-developed back-to-back glands and cribriform growth pattern. The cells showed moderate nuclear atypia with nuclear overlap, vesicular chromatin and an inconspicuous to the prominent eosinophilic nucleolus. The cytoplasm was slightly eosinophilic. There were numerous mitotic figures focally up to 6 mitoses/1HPF. Histology of the latest metastasis to the lung is depicted in Figure 1. Based on morphology, a diagnosis of metastatic well-differentiated endometrioid adenocarcinoma was considered. However, there was an inconsistency between the normally good prognosis of a low-grade endometrioid adenocarcinoma, part of the so-called WHO Type I tumors that are lowgrade, estrogen-related, often clinically indolent, endometrioid carcinomas [11] and the early metastases in this case. Additional immunohistochemistry (see Figure 2) was performed, the tumor cells were positive for PAX8 and partly for CK7 and negative for SATB2 and CK20, confirming their gynecological and not colorectal origin. Estrogen receptor (ER) and progesterone receptor (PR) were negative, as were the previous metastases and the primary tumor. There was diffuse GATA3 positivity but no TTF1 expression. CD10 showed focal luminal positivity. The tumor cells exhibited a wild-type p53 expression. Mismatch repair protein (MMR) expression was preserved (MMR proficient). 


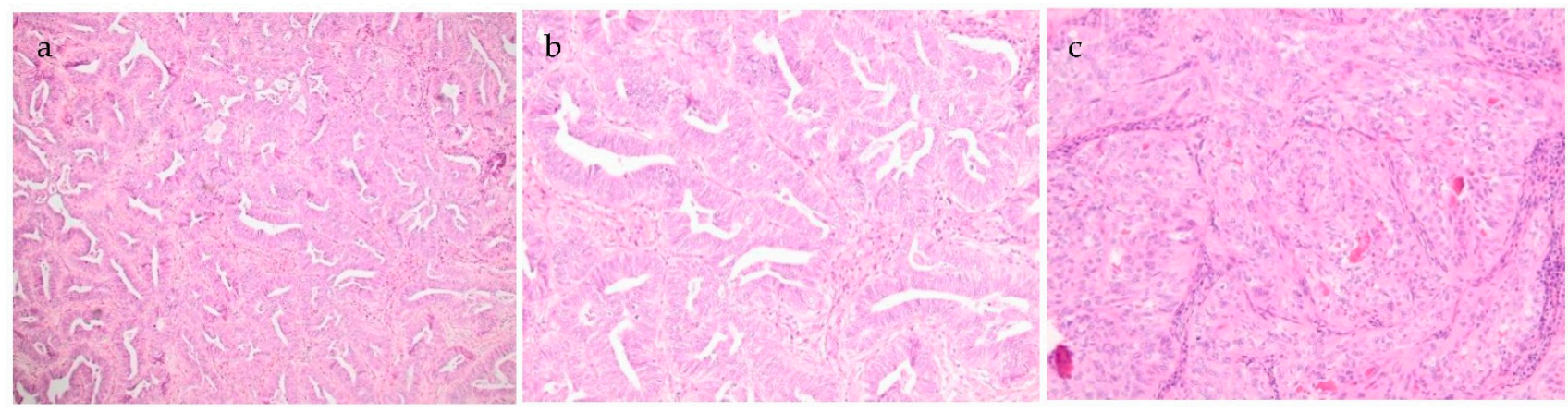

Figure 1. $(\mathbf{a}-\mathbf{c})$. Metastasis to the lung with glandular and ductular to focal solid growth pattern. Several eosinophilic intraluminal secretions are present (c). No high-grade atypia and low mitotic figures. (magnification: 40×, 100 $\times$, $100 \times$-HE staining).

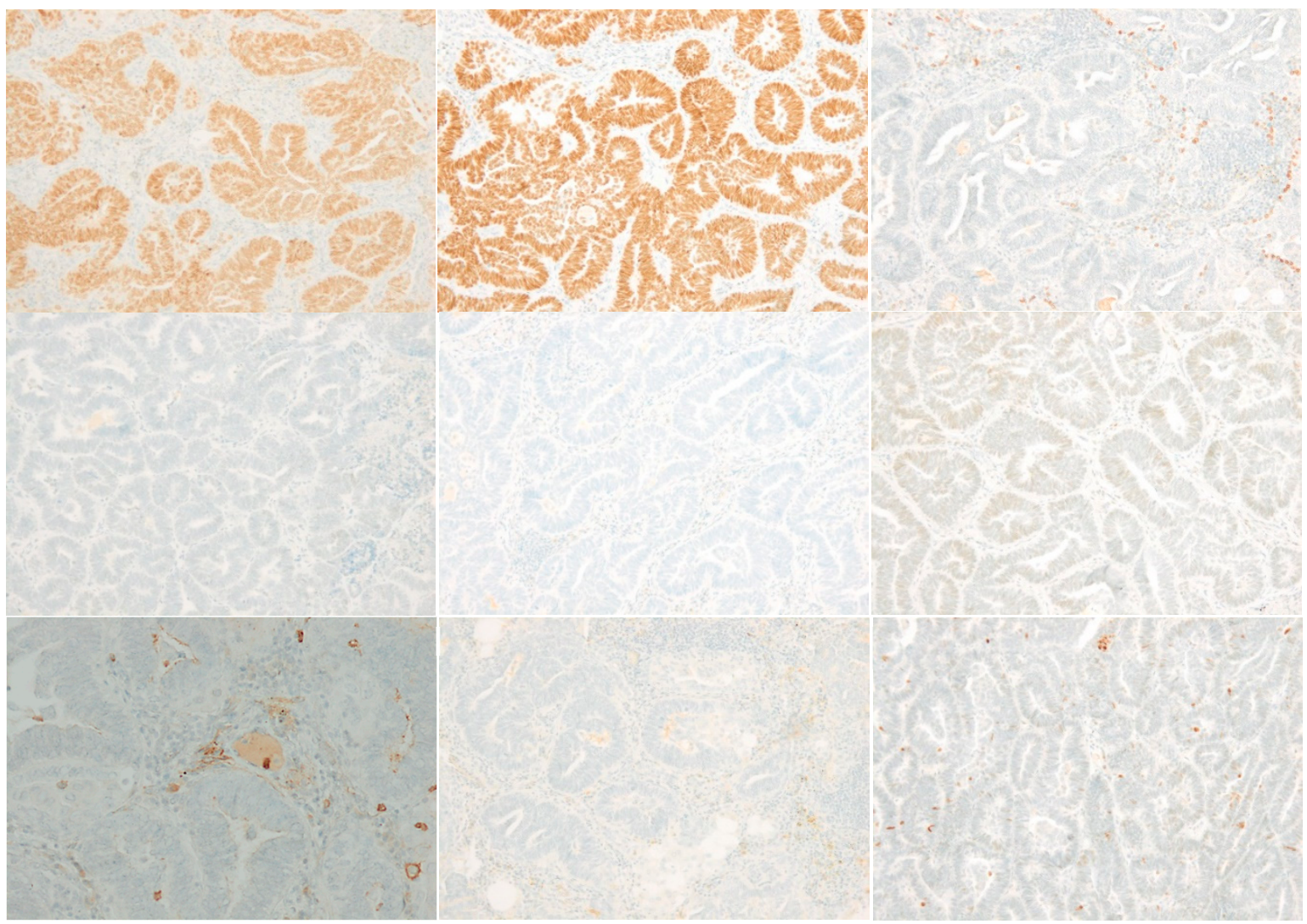

Figure 2. Diffuse positive staining for PAX8 and GATA3. TTF1, SATB2, estrogen receptor (ER) and progesterone receptor (PR) are negative (PR shows minor non-specific cytoplasmic background staining). CD10 focal luminal positivity. Calretinin negative; p53 wild type staining. (magnification: $100 \times$; immunohistochemistry).

The primary resection was reviewed and showed an endometrial lesion of $2.3 \mathrm{~cm}$ with invasion into the inner myometrium. It had a glandular growth pattern with some papillary snouting and loss of polarity. The cells were columnar with enlarged oval nuclei, coarse chromatin and an eosinophilic nucleolus. Mitotic activity was not noticeably increased. There was no necrosis, no perineural invasion and no lymphovascular space invasion. No squamous or mucinous differentiation was seen. The right fallopian tube showed a micropapillary serous borderline tumor. The morphology of this cystic papillary serous lesion with mild cytonuclear atypia did not resemble a possible metastasis of the endometrial mesonephric-like adenocarcinoma (with different architecture, different chromatin pattern, different aspect of nucleoli). The left tube and both ovaries were normal. 
With the morphology, supportive immunohistochemical profile and the history of early metastasis to a distant site, a diagnosis of a metastasis of a mesonephric-like adenocarcinoma was made. Additional molecular analysis showed a pathogenic KRAS c.38G > A variant (p.Gly13Asp) and two probable pathogenic variants of PTEN (c.388C > T and c. $634+2 \mathrm{~T}>\mathrm{G})$.

\subsection{Literature Search}

Twenty-seven articles were found with a total of 154 case reports of MLAs (see Tables 1-3). Of these, there were 115 cases of the uterine body and 39 cases of the ovary. There were 12 cases included that originate in the myometrium, 72 in the endometrium and 31 not specified. A total of 16 tumors had associated findings. The clinical findings are listed in Table 1 and the microscopic, immunohistochemical and molecular findings in Tables 2 and 3. For an overview of immunohistochemical findings, see Figure 3.

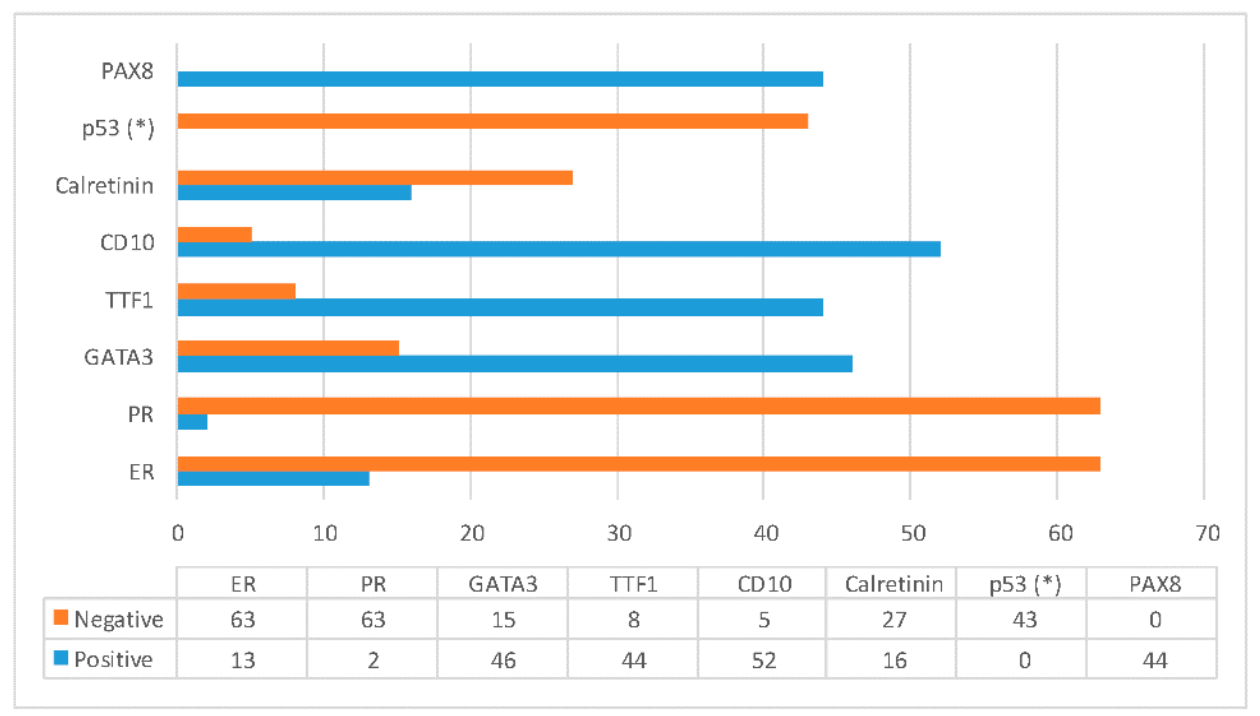

Figure 3. Summary of immunohistochemical findings. Only cases where information was available of individual markers are included. Estrogen receptor (ER) and progesterone receptor (PR) are nearly always negative, with PR as a more reliable negative marker. Mesonephric-like adenocarcinomas (MLAs) are characterized by staining with GATA binding protein 3 (GATA3) and/or thyroid transcription factor 1 (TTF1) with sometimes a reversed staining pattern. CD10 was predominantly positive in the tested cases but mostly focal. Calretinin is more often negative. All cases showed Paired box protein- 8 (PAX8) positivity and p53 wild type expression. $\left(^{*}\right)=$ negative for TP53 mutation. 
Table 1. Clinical findings.

\begin{tabular}{|c|c|c|c|c|c|c|c|c|c|c|}
\hline & Case & Age & Location & $\begin{array}{l}\text { Associated } \\
\text { Findings }\end{array}$ & $\begin{array}{l}\text { FIGO Stage } \\
\quad 2009\end{array}$ & Treatment & $\begin{array}{c}\text { Chemotherapy } \\
\text { Y/N }\end{array}$ & Radiation $\mathrm{Y} / \mathrm{N}$ & $\begin{array}{l}\text { Recurrence Y/N } \\
\quad \text { (Location } \\
\text { R/Treatment) }\end{array}$ & $\begin{array}{l}\text { Follow-Up } \\
\text { Time (mo) }\end{array}$ \\
\hline 1 & Yamamoto et al., 1995 [12] & 58 & Myometrium & $\begin{array}{c}\text { Cervical Gartner } \\
\text { duct cyst }\end{array}$ & $I A$ * & $\mathrm{TH}+\mathrm{BSO}$ & $N$ & $N$ & $\begin{array}{l}Y \text { (paraaortic and } \\
\text { mediastinal lymph } \\
\text { node metastases } \\
R / \text { cisplatinum }+ \\
\text { cyclophosphamide) }\end{array}$ & $8 D E D$ \\
\hline 2 & Ordi et al., 2001 [13] & 33 & Myometrium & None & $I A^{*}$ & $\begin{array}{c}\text { TH + BSO + } \\
\quad P L N D \\
\end{array}$ & $N$ & Y & $N$ & $8 N E D$ \\
\hline 3 & Montagut et al., 2003 [14] & 33 & Myometrium & None & $I B^{*}$ & $\begin{array}{c}\text { Myomectomy and } \\
\text { subsequent TH + } \\
\text { BSO + PLND + } \\
\text { PALND }\end{array}$ & $N$ & Y & $\begin{array}{c}Y \text { (peritoneal } \\
\text { carcinomatosis and } \\
\text { lung metastasis } \\
R / \text { carboplatin }+ \\
\text { paclitaxel) }\end{array}$ & $22 A W D$ \\
\hline 4 & Bague et al., 2004 [15] & 37 & Endometrium & $\begin{array}{c}\text { None } \\
\text { Diagnosed as } \\
\text { MMMT }\end{array}$ & IA * & $\mathrm{TH}+\mathrm{BSO}$ & $\mathrm{N}$ & $\mathrm{N}$ & $\mathrm{N}$ & 45 NED \\
\hline 5 & Marquette et al., 2006 [16] & 81 & $\begin{array}{l}\text { Myometrium to } \\
\text { endometrium }\end{array}$ & None & $I A$ * & $\begin{array}{c}T H+B S O+ \\
P L N D+P A L N D\end{array}$ & $N$ & $N$ & $N$ & $9 N E D$ \\
\hline 7 & Kenny et al., 2012 [18] & $N A$ & Myometrium & $\begin{array}{c}\text { Benign } \\
\text { mesonephric } \\
\text { remnants in the } \\
\text { cervix }\end{array}$ & IIIA & $\mathrm{TH}+\mathrm{BSO}$ & $N A$ & $N A$ & $N A$ & $N A$ \\
\hline 8 & Wu et al., 2014 [19] & 55 & Myometrium & None & $I B$ & $\begin{array}{l}T H+B S O+ \\
\quad P L N D\end{array}$ & $N$ & $N$ & $N$ & $7 N E D$ \\
\hline 9 & Wu et al., 2014 [19] & 62 & Myometrium & None & $I B$ & $\begin{array}{c}T H+B S O+ \\
P L N D\end{array}$ & $N$ & $N$ & $N$ & 1 NED \\
\hline 10 & Howitt et al., 2015 [20] & NA & NA & NA & NA & NA & NA & NA & NA & NA \\
\hline 11 & Kim et al., 2016 [21] & 66 & $\begin{array}{l}\text { Myometrium to } \\
\text { endometrium }\end{array}$ & Adenomyosis & IB & $\begin{array}{l}\mathrm{TH}+\mathrm{BSO}+ \\
\text { PLND }\end{array}$ & $\mathrm{N}$ & $\mathrm{N}$ & $\mathrm{N}$ & 2 NED \\
\hline
\end{tabular}


Table 1. Cont.

\begin{tabular}{|c|c|c|c|c|c|c|c|c|c|c|}
\hline & Case & Age & Location & $\begin{array}{l}\text { Associated } \\
\text { Findings }\end{array}$ & $\begin{array}{c}\text { FIGO Stage } \\
\quad 2009\end{array}$ & Treatment & $\begin{array}{c}\text { Chemotherapy } \\
\text { Y/N }\end{array}$ & Radiation Y/N & $\begin{array}{l}\text { Recurrence Y/N } \\
\text { (Location } \\
\text { R/Treatment) }\end{array}$ & $\begin{array}{l}\text { Follow-Up } \\
\text { Time (mo) }\end{array}$ \\
\hline $12-23$ & McFarland et al., 2016 [22] & $42-72$ & $\begin{array}{c}7 \text { uterine corpus } \\
5 \text { ovarian }\end{array}$ & $\begin{array}{c}\text { Endometriosis } \\
\text { in } 3 \text { of } 5 \\
\text { (ovarian cases) } \\
\text { Adenomyosis } \\
(1 / 7)\end{array}$ & $\begin{array}{l}\text { IA(3 cases), IB(1 } \\
\text { case), IIIC(1 } \\
\text { case })\end{array}$ & NA & NA & $\mathrm{NA}$ & $\begin{array}{l}\mathrm{N} \text { (IA, IB cases) } \mathrm{Y} \\
\text { (IIIC case) }\end{array}$ & $\begin{array}{c}18 \text { (IA, IB cases) } \\
56 \text { (IIIC case) }\end{array}$ \\
\hline 24 & Ando et al., 2017 [23] & 61 & Myometrium & Adenomyosis & $I B$ & $T H+B S O$ & $\begin{array}{c}Y \text { carboplatin }+ \\
\text { paclitaxel }\end{array}$ & $N$ & $N$ & $9 N E D$ \\
\hline 25 & Kim et al., 2018 [24] & 37 & $\begin{array}{l}\text { Endometrium to } \\
\text { myometrium }\end{array}$ & None & IB & $\mathrm{TH}+\mathrm{BSO}$ & NA & NA & NA & NA \\
\hline 26 & Zhang et al., 2019 [25] & 63 & $\begin{array}{l}\text { Endometrium to } \\
\text { myometrium }\end{array}$ & None & IB & $\begin{array}{c}\text { TH + BSO+ } \\
\text { PLND + PALND }\end{array}$ & $\mathrm{N}$ & Y & $\mathrm{N}$ & 31 NED \\
\hline 27 & Zhang et al., 2019 [25] & 57 & Myometrium & None & IIIB & $\begin{array}{c}\mathrm{TH}+\mathrm{BSO}+ \\
\text { PLND }\end{array}$ & Y & $\mathrm{N}$ & NA & NA \\
\hline 28 & Chapel et al., 2018 [3] & 80 & Ovary & $\begin{array}{c}\text { Serous } \\
\text { borderline } \\
\text { tumor and } \\
\text { low-grade } \\
\text { serous } \\
\text { carcinoma }\end{array}$ & not reported & $\begin{array}{c}\mathrm{TH}+\mathrm{BSO}+ \\
\text { omentectomy + } \\
\text { tumor } \\
\text { debulking }\end{array}$ & $\begin{array}{c}\text { Y Neoadjuvant } \\
\text { carboplatin + } \\
\text { paclitaxel }\end{array}$ & $\mathrm{N}$ & $\mathrm{N}$ & 3 NED \\
\hline 29 & Patel et al., 2018 [26] & 71 & $\begin{array}{l}\text { Endometrium to } \\
\text { myometrium }\end{array}$ & None & IB & $\mathrm{TH}+\mathrm{BSO}$ & NA & NA & NA & NA \\
\hline 30 & Pors et al., 2018 [27] & 65 & Endometrium & None & IVB & NA & $\mathrm{NA}$ & $\mathrm{NA}$ & NA & NA \\
\hline 31 & Pors et al., 2018 [27] & 31 & Endometrium & None & IIIA & $\mathrm{NA}$ & $\mathrm{NA}$ & $\mathrm{NA}$ & NA & $\mathrm{NA}$ \\
\hline 32 & Pors et al., 2018 [27] & 75 & Endometrium & None & IB & NA & NA & NA & NA & NA \\
\hline 33 & Pors et al., 2018 [27] & 91 & Endometrium & None & IIIA & NA & NA & NA & NA & NA \\
\hline 34 & Pors et al., 2018 [27] & 67 & Ovary & None & IC & $\mathrm{NA}$ & $\mathrm{NA}$ & $\mathrm{NA}$ & NA & NA \\
\hline 35 & Na et al., 2019 [9] & 58 & $\begin{array}{l}\text { Endometrium to } \\
\text { myometrium }\end{array}$ & None & IIIB & $\begin{array}{c}\text { TH + BSO + } \\
\text { PLND + PALND }\end{array}$ & $\begin{array}{c}\text { Y carboplatin }+ \\
\text { paclitaxel }\end{array}$ & Y & $\begin{array}{c}\mathrm{Y} \text { (lung } \\
\mathrm{R} / \text { carboplatin }+ \\
\text { paclitaxel }\end{array}$ & 56 AWD \\
\hline 36 & Na et al., 2019 [9] & 55 & $\begin{array}{l}\text { Endometrium to } \\
\text { myometrium }\end{array}$ & None & IVB & $\mathrm{TH}+\mathrm{BSO}$ & $\begin{array}{c}\text { Y carboplatin }+ \\
\text { paclitaxel }\end{array}$ & Y & $\begin{array}{l}\text { Y (carboplatin }+ \\
\text { paclitaxel) }\end{array}$ & $21 \mathrm{AWD}$ \\
\hline 37 & Na et al., 2019 [9] & 54 & $\begin{array}{l}\text { Endometrium to } \\
\text { myometrium }\end{array}$ & None & IIIB & $\mathrm{TH}+\mathrm{BSO}$ & $\begin{array}{c}\text { Y carboplatin }+ \\
\text { paclitaxel }\end{array}$ & Y & $\begin{array}{c}\mathrm{Y} \text { (lung } \\
\mathrm{R} / \text { doxorubicin }+ \\
\text { cisplatin) }\end{array}$ & 20 AWD \\
\hline
\end{tabular}


Table 1. Cont.

\begin{tabular}{|c|c|c|c|c|c|c|c|c|c|c|}
\hline & Case & Age & Location & $\begin{array}{l}\text { Associated } \\
\text { Findings }\end{array}$ & $\begin{array}{l}\text { FIGO Stage } \\
\quad 2009\end{array}$ & Treatment & $\begin{array}{c}\text { Chemotherapy } \\
\text { Y/N }\end{array}$ & Radiation Y/N & $\begin{array}{l}\text { Recurrence Y/N } \\
\text { (Location } \\
\text { R/Treatment) }\end{array}$ & $\begin{array}{l}\text { Follow-Up } \\
\text { Time (mo) }\end{array}$ \\
\hline 38 & Na et al., 2019 [9] & 60 & $\begin{array}{l}\text { Endometrium to } \\
\text { myometrium }\end{array}$ & None & IA & $\begin{array}{c}\text { TH + BSO + } \\
\text { PLND + PALND }\end{array}$ & Y & $\mathrm{N}$ & $\begin{array}{c}\mathrm{Y} \text { (lung } \\
\mathrm{R} / \text { carboplatin }+ \\
\text { paclitaxel) }\end{array}$ & 14 AWD \\
\hline 38 & Na et al., 2019 [9] & 53 & $\begin{array}{l}\text { Endometrium to } \\
\text { myometrium }\end{array}$ & None & IA & $\begin{array}{c}\text { TH + BSO + } \\
\text { PLND + PALND }\end{array}$ & $\mathrm{N}$ & $\mathrm{N}$ & $\mathrm{N}$ & 12 NED \\
\hline 40 & Na et al., 2019 [9] & 57 & Myometrium & None & IIIC & $\begin{array}{c}\text { TH + BSO + } \\
\text { PLND + PALND }\end{array}$ & $\begin{array}{l}\text { Y carboplatin + } \\
\text { paclitaxel }\end{array}$ & $\mathrm{N}$ & $\begin{array}{c}\mathrm{Y} \text { (lung } \\
\mathrm{R} / \text { carboplatin }+ \\
\text { paclitaxel) }\end{array}$ & 13 AWD \\
\hline 41 & Na et al., 2019 [9] & 70 & $\begin{array}{l}\text { Endometrium to } \\
\text { myometrium }\end{array}$ & None & IB & $\begin{array}{c}\text { TH + BSO + } \\
\text { PLND + PALND }\end{array}$ & $\mathrm{N}$ & Y & $\mathrm{N}$ & 10 NED \\
\hline 42 & Na et al., 2019 [9] & 61 & $\begin{array}{l}\text { Endometrium to } \\
\text { myometrium }\end{array}$ & None & IB & $\begin{array}{c}\text { TH + BSO + } \\
\text { PLND + PALND }\end{array}$ & $\begin{array}{l}\text { Y carboplatin + } \\
\text { paclitaxel }\end{array}$ & $\mathrm{N}$ & $\mathrm{N}$ & 7 NED \\
\hline 43 & Na et al., 2019 [9] & 65 & $\begin{array}{l}\text { Endometrium to } \\
\text { myometrium }\end{array}$ & None & IB & $\begin{array}{c}\text { TH + BSO + } \\
\text { PLND + PALND }\end{array}$ & $\mathrm{N}$ & $\mathrm{N}$ & $\mathrm{N}$ & $6 \mathrm{NED}$ \\
\hline 44 & Na et al., 2019 [9] & 59 & $\begin{array}{l}\text { Endometrium to } \\
\text { myometrium }\end{array}$ & None & IA & $\begin{array}{c}\text { TH + BSO + } \\
\text { PLND + PALND }\end{array}$ & $\mathrm{N}$ & $\mathrm{N}$ & $\mathrm{N}$ & 11 NED \\
\hline 46 & Yano et al., 2019 [28] & 32 & $\begin{array}{l}\text { Endometrium to } \\
\text { myometrium }\end{array}$ & $\begin{array}{l}\text { Low grade } \\
\text { endometrioid } \\
\text { carcinoma }\end{array}$ & IA & $\begin{array}{c}\mathrm{TH}+\mathrm{BSO}+ \\
\text { omentectomy }\end{array}$ & $\begin{array}{l}\text { medroxyprogesterone } \\
\text { acetate }\end{array}$ & $\mathrm{N}$ & Y 6 year later & NA \\
\hline 47 & Kolin et al., 2019 [8] & 64 & $\begin{array}{l}\text { Endometrium to } \\
\text { myometrium }\end{array}$ & None & IB & $\begin{array}{l}\text { TH + PLND + } \\
\text { omentectomy }\end{array}$ & NA & NA & $\begin{array}{c}\text { Y local (3y) lung } \\
\text { metastases (12y } \\
\text { R/carboplatin/taxol) }\end{array}$ & 150 AWD \\
\hline 48 & Kolin et al., 2019 [8] & 57 & $\begin{array}{l}\text { Endometrium to } \\
\text { myometrium }\end{array}$ & None & IA & NA & NA & NA & $\mathrm{N}$ & 18 NED \\
\hline 49 & Kolin et al., 2019 [8] & 58 & $\begin{array}{l}\text { Endometrium to } \\
\text { myometrium }\end{array}$ & None & IVB & NA & NA & NA & Y (local) & $30 \mathrm{AWD}$ \\
\hline 50 & Kolin et al., 2019 [8] & 62 & $\begin{array}{l}\text { Endometrium to } \\
\text { myometrium }\end{array}$ & None & IIIC & NA & NA & NA & Y (lung) & 100 DOD \\
\hline
\end{tabular}


Table 1. Cont.

\begin{tabular}{|c|c|c|c|c|c|c|c|c|c|c|}
\hline & Case & Age & Location & Associated Findings & $\begin{array}{l}\text { FIGO Stage } \\
\quad 2009\end{array}$ & Treatment & $\begin{array}{c}\text { Chemotherapy } \\
\text { Y/N }\end{array}$ & Radiation $\mathrm{Y} / \mathrm{N}$ & $\begin{array}{l}\text { Recurrence Y/N } \\
\text { (Location } \\
\text { R/Treatment) }\end{array}$ & $\begin{array}{l}\text { Follow-Up } \\
\text { Time (mo) }\end{array}$ \\
\hline 51 & Yamamoto et al., 2019 [29] & 70 & $\begin{array}{l}\text { Endometrium to } \\
\text { myometrium }\end{array}$ & None & IA & $\mathrm{TH}$ & NA & NA & Y (lung (5y)) & NA \\
\hline 52 & McCluggage et al., 2020 [4] & 61 & Ovary & $\begin{array}{l}\text { Serous borderline } \\
\text { tumor (low-grade } \\
\text { serous carcinoma in } \\
\text { extraovarian tissues) }\end{array}$ & IIIA1 & NA & $\begin{array}{c}\text { Y carboplatin + } \\
\text { paclitaxel }\end{array}$ & $\mathrm{N}$ & NA & NA \\
\hline 53 & McCluggage et al., 2020 [4] & 66 & Ovary & $\begin{array}{c}\text { Borderline } \\
\text { endometrioid } \\
\text { adenofibroma }\end{array}$ & NA & NA & NA & NA & NA & NA \\
\hline 54 & McCluggage et al., 2020 [4] & 77 & Ovary & $\begin{array}{l}\text { Endometriosis, mixed } \\
\text { serous and mucinous } \\
\text { cystadenoma }\end{array}$ & NA & NA & NA & NA & NA & NA \\
\hline 55 & McCluggage et al., 2020 [4] & 50 & Ovary & None & NA & NA & NA & NA & NA & NA \\
\hline 56 & McCluggage et al., 2020 [4] & 73 & Ovary & Serous cystadenoma & NA & NA & NA & NA & NA & NA \\
\hline 57 & Dundr et al., 2020 [30] & 61 & Ovary & $\begin{array}{l}\text { Serous borderline } \\
\text { tumor }\end{array}$ & IV & $\begin{array}{c}\mathrm{TH}+\mathrm{BSA}+ \\
\text { resection of liver } \\
\text { metastases and } \\
\text { the diaphragm, } \\
\text { total } \\
\text { omentectomy, } \\
\text { appendectomy } \\
\text { and a resection } \\
\text { of an umbilical } \\
\text { metastasis }\end{array}$ & $\begin{array}{c}\text { Y Neoadjuvant: } \\
\text { carboplatin + } \\
\text { paclitaxel } \\
\text { Postoperative: } \\
\text { carboplatin + } \\
\text { paclitaxel + } \\
\text { bevacizumab }\end{array}$ & $\mathrm{N}$ & $\mathrm{N}$ & 12 NED \\
\hline 58 & Seay et al., 2020 [31] & 67 & Ovary & Endometriosis & IA & $\begin{array}{c}\mathrm{TH}+\mathrm{BSO}+ \\
\text { PLND + } \\
\text { omentectomy }\end{array}$ & $\mathrm{N}$ & $\mathrm{N}$ & $\begin{array}{c}\text { Y (abdominal } 18 \text { mo } \\
\text { R/carboplatin+ } \\
\text { paclitaxel } \\
\text { +bevacizumab) }\end{array}$ & 18 AWD \\
\hline
\end{tabular}


Table 1. Cont.

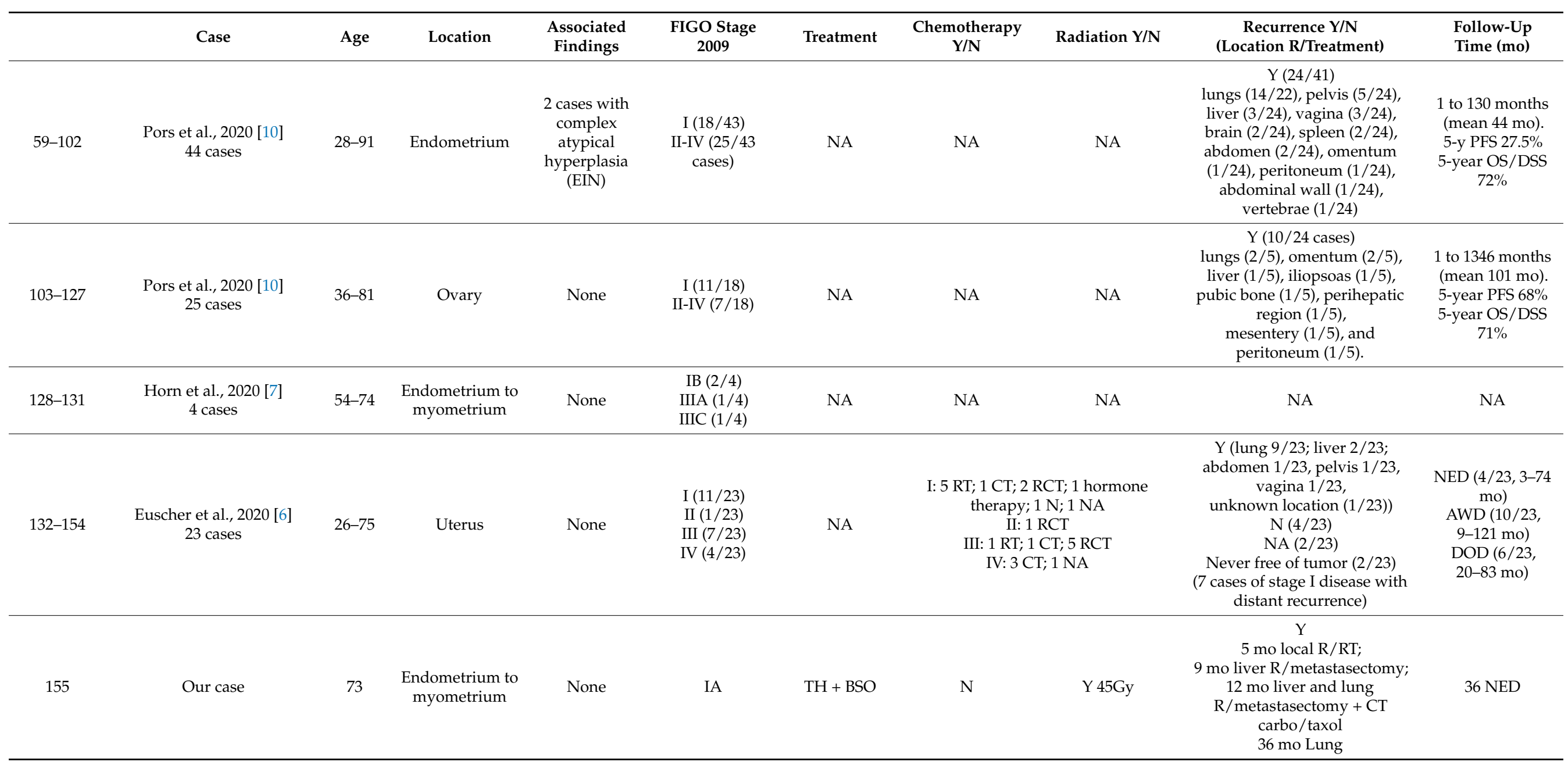

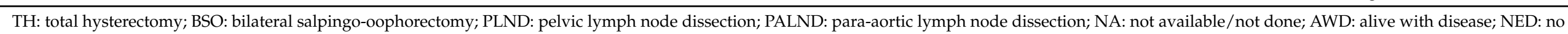
evidence of disease; DOD: died of disease; RT: radiation therapy; CT: chemotherapy; RCT: radiation + chemotherapy; mo: month(s); Y: yes; N: no. * = FIGO stage adjusted to 2009 classification. 
Table 2. Morphologic, immunohistochemical and molecular findings.

\begin{tabular}{|c|c|c|c|c|c|c|c|c|c|c|}
\hline & Case & Tubular & Papillary & Glandular & Solid & Spindle Cell & Retform & $\begin{array}{l}\text { Eosinophilic } \\
\text { Secretions }\end{array}$ & Atypia & Other \\
\hline 1 & Yamamoto et al., 1995 [12] & $x$ & $x$ & & $x$ & $x$ & & & Severe. & Frequent mitotic figures. \\
\hline 2 & Ordi et al., 2001 [13] & $x$ & $x$ & $x$ & $x$ & $x$ & $x$ & $x$ & Moderate. & Mitotic index $3 / H P F$ \\
\hline 3 & Montagut et al., 2003 [14] & $x$ & & $x$ & & $x$ & $x$ & $x$ & $N A$ & $N A$ \\
\hline 4 & Bague et al., 2004 [15] & & $\mathrm{x}$ & $x$ & $\mathrm{x}$ & $x$ & & $x$ & & \\
\hline 5 & Marquette et al., 2006 [16] & $x$ & $x$ & $x$ & & & $x$ & $x$ & & \\
\hline 7 & Kenny et al., 2012 [18] & $x$ & $x$ & $x$ & $x$ & $x$ & & $x$ & Focally severe. & $\begin{array}{l}\text { Sex cord like } \\
\text { Focal mitotic activity }\end{array}$ \\
\hline $8-9$ & $W u$ et al., 2014 [19] 2 cases & $x$ & & $x$ & $x$ & $x$ & $x$ & $x$ & & Sex cord like \\
\hline 10 & Howitt et al., 2015 [20] & & & & & & & & & \\
\hline 11 & Kim et al., 2016 [21] & $\mathrm{x}$ & $\mathrm{x}$ & $\mathrm{x}$ & & & $\mathrm{x}$ & $\mathrm{x}$ & Mild. & $\begin{array}{l}\text { Glomeruloid } \\
\text { Mitotic index } \\
>10 / 10 \mathrm{HPFs}\end{array}$ \\
\hline 24 & Ando et al., 2017 [23] & $x$ & $x$ & $x$ & $x$ & & & $x$ & $\begin{array}{l}\text { Mild. } \\
\text { More atypical } \\
\text { component in the } \\
\text { periphery. }\end{array}$ & Occasional mitotic figures. \\
\hline 25 & Kim et al., 2018 [24] & $\mathrm{x}$ & & $\mathrm{x}$ & & & & $x$ & $\begin{array}{l}\text { Severe, yet } \\
\text { monomorphic } \\
\text { nuclei }\end{array}$ & \\
\hline 26 & Zhang et al., 2019 [25] & $\mathrm{x}$ & $\mathrm{x}$ & & $\mathrm{x}$ & $\mathrm{x}$ & & $\mathrm{x}$ & $\begin{array}{l}\text { Bland cuboidal } \\
\text { cells }\end{array}$ & \\
\hline 27 & Zhang et al., 2019 [25] & $\mathrm{x}$ & $\mathrm{x}$ & & $\mathrm{x}$ & & $x$ & $x$ & & \\
\hline
\end{tabular}


Table 2. Cont.

\begin{tabular}{|c|c|c|c|c|c|c|c|c|c|c|}
\hline & Case & Tubular & Papillary & Glandular & Solid & Spindle Cell & Retform & $\begin{array}{l}\text { Eosinophilic } \\
\text { Secretions }\end{array}$ & Atypia & Other \\
\hline 28 & Chapel et al., 2018 [3] & $\mathrm{x}$ & & & $\mathrm{x}$ & & & $\mathrm{x}$ & $\begin{array}{l}\text { Mild to } \\
\text { moderate. }\end{array}$ & $\begin{array}{l}\text { Psammomatous } \\
\text { calcifications focally } \\
\text { Mitotic index 1-2/10 } \\
\text { HPFs. }\end{array}$ \\
\hline 29 & Patel et al., 2018 [26] & $x$ & $x$ & $\mathrm{x}$ & & & & $x$ & Mild & $\begin{array}{l}\text { Prominent stromal } \\
\text { hyalinization } \\
\text { Mitotic activity was } \\
\text { relatively low level } \\
\text { without atypical mitotic } \\
\text { figures }\end{array}$ \\
\hline
\end{tabular}

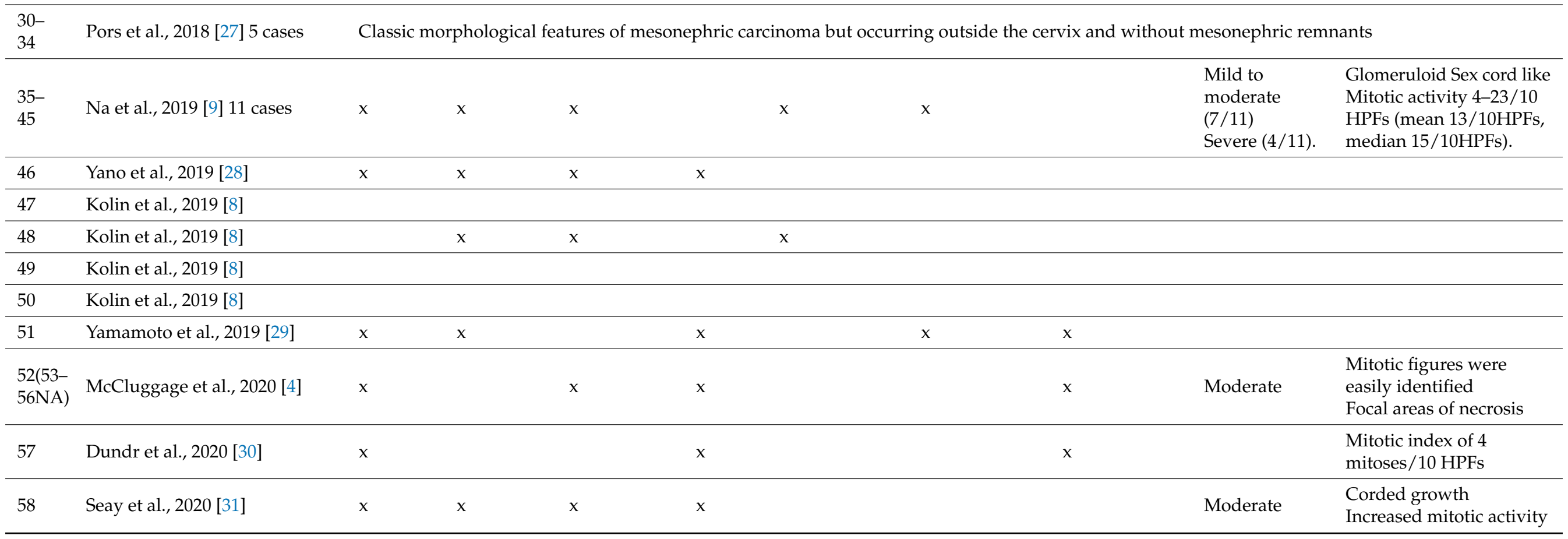


Table 2. Cont.

\begin{tabular}{|c|c|c|c|c|c|c|c|c|c|c|}
\hline & Case & Tubular & Papillary & Glandular & Solid & Spindle Cell & Retform & $\begin{array}{l}\text { Eosinophilic } \\
\text { Secretions }\end{array}$ & Atypia & Other \\
\hline $\begin{array}{l}59- \\
127\end{array}$ & $\begin{array}{l}\text { Pors et al., } 2020 \text { [10] } \\
44+25 \text { cases }\end{array}$ & \multicolumn{9}{|c|}{ Classic morphological features of mesonephric carcinoma but occurring outside the cervix and without mesonephric remnants. } \\
\hline $\begin{array}{l}128- \\
131\end{array}$ & $\begin{array}{l}\text { Horn et al., } 2020[7] \\
4 \text { cases }\end{array}$ & $\mathrm{x}$ & $x$ & $\mathrm{x}$ & $\mathrm{x}$ & & & & Low grade & $\begin{array}{l}\text { Mean mitotic index of } \\
26.5 / 10 \text { HPFs (range } \\
21-33 \text { ). }\end{array}$ \\
\hline $\begin{array}{l}132- \\
154\end{array}$ & $\begin{array}{l}\text { Euscher et al., } 2020 \text { [6] } \\
23 \text { cases }\end{array}$ & $x$ & $x$ & $\mathrm{x}$ & $\mathrm{x}$ & $x$ & $x$ & $\mathrm{x}$ & $\begin{array}{l}\text { Mild to } \\
\text { moderate } \\
\text { Focally } \\
\text { marked }\end{array}$ & $\begin{array}{l}\text { Cords / trabeculae } \\
\text { Sieve like } \\
\text { Mitotic index } \\
\text { 3-28/10 HPFs (median: } \\
10)\end{array}$ \\
\hline 155 & Our case & $x$ & & $\mathrm{x}$ & & & & & Moderate & Mitotic index 2-6/1HPF. \\
\hline
\end{tabular}

Italics: located in the myometrium. NA: not available; HPF: High power field; $x$ : present; +: positive; -: negative. 
Table 3. Morphologic, immunohistochemical and molecular findings.

\begin{tabular}{|c|c|c|c|c|c|c|c|}
\hline & \multirow{2}{*}{ Case } & \multicolumn{5}{|c|}{ Immunohistochemistry (IHC) } & \multirow{2}{*}{ Molecular } \\
\hline & & ER/PR & GATA3 & TTF-1 & CD10 & Other/Remarks & \\
\hline 1 & Yamamoto et al., 1995 [12] & $N A$ & $N A$ & $N A$ & $N A$ & I & $N A$ \\
\hline 2 & Ordi et al., 2001 [13] & $-1-$ & $N A$ & $N A$ & + & $\begin{array}{l}\text { P53 wild type } \\
\text { Inhibin - }\end{array}$ & $N A$ \\
\hline 3 & Montagut et al., 2003 [14] & $-1-$ & $N A$ & $N A$ & + & P53 wild type & $N A$ \\
\hline 4 & Bague et al., 2004 [15] & NA & NA & NA & Not specified & $\begin{array}{l}\text { Case diagnosed as } \\
\text { MMMT }\end{array}$ & NA \\
\hline 6 & Wani et al., 2008 [17] & $-/-$ & $N A$ & $N A$ & +luminal & $\begin{array}{l}\text { Calretinin }+ \\
\text { P53 wild type } \\
\text { HNF1b- }\end{array}$ & $N A$ \\
\hline 7 & Kenny et al., 2012 [18] & Not specified (2/8+) & $N A$ & Not specified $(3 / 5+)$ & Not specified $(6 / 8+)$ & $\begin{array}{l}\text { Report of } 8 \text { cases, } 7 \\
\text { cervical, } 1 \text { uterine body; } \\
\text { IHC is not separately } \\
\text { reported. }\end{array}$ & $N A$ \\
\hline $8-9$ & $\begin{array}{l}\text { Wu et al., } 2014 \text { [19] } \\
2 \text { cases }\end{array}$ & $-1-$ & $N A$ & $N A$ & + & Calretinin + & $N A$ \\
\hline 11 & Kim et al., 2016 [21] & $-1-$ & NA & + & + luminal & $\begin{array}{l}\text { Calretinin - } \\
\text { Inhibin - } \\
\text { P53 wild type }\end{array}$ & NA \\
\hline $12-23$ & $\begin{array}{l}\text { McFarland et al., } 2016 \text { [22] } \\
12 \text { cases } \\
+ \text { Mirkovic et al., } 2018 \text { [1] for } \\
\text { molecular analysis ( } 7 \text { cases } \\
\text { of McFarland et al.) }\end{array}$ & $-/-(12 / 12)$ & $\begin{array}{l}+(3 / 12) \\
-(8 / 12)\end{array}$ & $\begin{array}{l}+(11 / 12) \\
-(1 / 12)\end{array}$ & $\begin{array}{l}+(7 / 9) \\
-(2 / 9)\end{array}$ & $\begin{array}{l}\text { P53 wild type } \\
\text { Calretinin }+(3 / 6) \\
\text { HNF1b }-(8 / 10) \\
\text { NapsinA }-(9 / 11)\end{array}$ & $\begin{array}{l}\text { KRAS mutations } 7 / 7 \\
\text { - G12D }(4 / 7) \\
\text { PIK3CA activating mutations ( }(3 / 7) \text {. } \\
\text { There were no alterations in PTEN, ARID1A, } \\
\text { or TP53 in any of the tumors. } \\
\text { CNV: } 1 \text { q gain }(5 / 7) \text {, accompanied by } 1 \mathrm{p} \text { loss } \\
\text { in } 2 \text { cases. } \\
\text { Chromosome } 10 \text { gain }(4 / 7), \text { which was } \\
\text { accompanied by gain of chromosome } 12 \text { in } 3 \\
\text { cases. }\end{array}$ \\
\hline
\end{tabular}


Table 3. Cont.

\begin{tabular}{|c|c|c|c|c|c|c|c|}
\hline & \multirow{2}{*}{ Case } & \multicolumn{5}{|c|}{ Immunohistochemistry (IHC) } & \multirow{2}{*}{ Molecular } \\
\hline & & ER/PR & GATA3 & TTF-1 & CD10 & Other/Remarks & \\
\hline 24 & Ando et al., 2017 [23] & $+(<1 \%) /-$ & + & + & + luminal & $\begin{array}{l}\text { Calretinin }+ \text { focal } \\
\text { WT1 }- \\
\text { Inhibin }- \\
\text { NapsinA }+(<1 \%) \\
\text { P53 wild type }\end{array}$ & $N A$ \\
\hline 25 & Kim et al., 2018 [24] & $-1-$ & + & NA & + & I & NA \\
\hline 26 & Zhang et al., 2019 [25] & $-1-$ & + & NA & + luminal & $\begin{array}{l}\text { Calretinin + focal } \\
\text { WT1 + focal }\end{array}$ & NA \\
\hline 27 & Zhang et al., 2019 [25] & $-1-$ & + & - & NA & $\begin{array}{l}\text { WT1 - } \\
\text { NapsinA - } \\
\text { P53 wild type } \\
\text { P16 patchy }\end{array}$ & NA \\
\hline 28 & Chapel et al., 2018 [3] & $-1-$ & + & + & + luminal & $\begin{array}{l}\text { Calretinin - } \\
\text { P53 wild type } \\
\text { P16 patchy } \\
\text { WT1 - } \\
\text { Inhibin + focal } \\
\text { P63 + focal } \\
\text { Thyroglobulin - } \\
\text { CK20 - } \\
\text { PTH - } \\
\text { Chromogranin - } \\
\text { Synaptophysin - }\end{array}$ & $\begin{array}{l}\text { NRAS Q61R } \\
\text { additional mutations in the tumor } \\
\text { suppressor genes } B C O R \text { or } A M E R 1 \\
\text { CNV: } \\
1 \text { q gain, 18p gain, 1p loss, 18q loss, } 22 \text { loss. }\end{array}$ \\
\hline 29 & Patel et al., 2018 [26] & $+($ weak focal $<5 \%) /-$ & + & + & NA & $\begin{array}{l}\text { Beta-catenin nuclear } \\
\text { Inverse staining of } \\
\text { GATA3/TTF1 }\end{array}$ & KRAS G12A \\
\hline $30-34$ & $\begin{array}{l}\text { Pors et al., } 2018 \text { [27] } \\
5 \text { cases }\end{array}$ & $\begin{array}{l}+/ \mathrm{ND}(2 / 5) \\
-/ \mathrm{ND}(3 / 5)\end{array}$ & $+(5 / 5)$ & $+(5 / 5)$ & + luminal $(4 / 5)$ & $\begin{array}{l}\text { Inverse staining of } \\
\text { GATA3/TTF1 } \\
\text { Calretinin - }\end{array}$ & NA \\
\hline
\end{tabular}


Table 3. Cont

\begin{tabular}{|c|c|c|c|c|c|c|c|}
\hline & \multirow{2}{*}{ Case } & \multicolumn{5}{|c|}{ Immunohistochemistry (IHC) } & \multirow{2}{*}{ Molecular } \\
\hline & & ER/PR & GATA3 & TTF-1 & CD10 & Other/Remarks & \\
\hline $35-45$ & $\begin{array}{l}\text { Na et al., } 2019[9] \\
11 \text { cases }\end{array}$ & $-/-(11 / 11)$ & $+(11 / 11)$ & NA & + luminal (11/11) & $\begin{array}{l}\text { P53 wild type } \\
\text { Preserved PTEN } \\
\text { Calretinin }+(3 / 11)\end{array}$ & $\begin{array}{l}\text { 11 cases + from } 1 \text { case a metastasis. } \\
\text { KRAS mutation }(10 / 12) \\
\bullet \quad \text { G12V }(6 / 10) \\
-\quad \text { G12C }(2 / 10) \\
\text { ARID1A mutation }(9 / 12) \\
-\quad \text { T294P }(6 / 9) \\
-\quad \text { Q288P }(2 / 9) \\
\text { 1q gain }(11 / 12) 2 \text { of the cases with 1q gain } \\
\text { also had loss of } 1 \mathrm{p} . \\
\text { 9p gain }(7 / 12), 20 \mathrm{q} \text { gain }(7 / 12), \\
\text { 12q gain }(6 / 12), 6 \mathrm{q} \text { gain }(4 / 12), 10 \mathrm{q} \text { gain } \\
(4 / 12), 3 \mathrm{q} \text { loss }(3 / 12), 5 \mathrm{p} \text { gain }(3 / 12), 7 \mathrm{q} \text { gain } \\
(3 / 12), 19 \mathrm{p} \text { gain }(3 / 12), \text { and gain of } \\
\text { chromosome } 2(3 / 12) . \\
\text { Gain of } 10 \mathrm{q} \text { was detected exclusively in } 3 \\
\text { cases with metastasis. } \\
\text { Additional PTEN mutation (D268E) in } \\
\text { metastatic tumor only. }\end{array}$ \\
\hline 46 & Yano et al., 2019 [28] & $-1-$ & + focal & + focal & + focal & $\begin{array}{l}\text { P53 wild type } \\
\text { CA125 strong } \\
\text { P16 focal } \\
\text { Calretinin - } \\
\text { HNF1b - } \\
\text { NapsinA - } \\
\text { AR - } \\
\text { WT1 - }\end{array}$ & KRAS G12A \\
\hline 47 & Kolin et al., 2019 [8] & $+($ patchy, weak $) /-$ & + focal & + & + luminal & $\begin{array}{l}\text { Synaptophysin - } \\
\text { Chromogranin - } \\
\text { P63 - }\end{array}$ & $\begin{array}{l}\text { KRAS G12A } \\
\text { 1p loss, 1q gain, 10p gain, 10q loss, } 21 \mathrm{q} \text { loss }\end{array}$ \\
\hline 48 & Kolin et al., 2019 [8] & +/+ heterogenous & - & + & + luminal & P53 wild type & $\begin{array}{l}\text { KRAS G12V } \\
1 \mathrm{p} \text { loss, 1q gain, 4p loss, 4q loss, 11p loss, 11q } \\
\text { loss, 21q loss }\end{array}$ \\
\hline 49 & Kolin et al., 2019 [8] & $-1-$ & - & + patchy & + luminal & $\begin{array}{l}\text { P16 patchy } \\
\text { NapsinA focal }+\end{array}$ & $\begin{array}{l}\text { KRAS G12D } \\
\text { PIK3R1 E451del } \\
\text { 1q gain, 11p loss, 11q loss, 13q loss, 17p loss, } \\
\text { 22q loss }\end{array}$ \\
\hline
\end{tabular}


Table 3. Cont.

\begin{tabular}{|c|c|c|c|c|c|c|c|}
\hline & \multirow{2}{*}{ Case } & \multicolumn{5}{|c|}{ Immunohistochemistry (IHC) } & \multirow{2}{*}{ Molecular } \\
\hline & & ER/PR & GATA3 & TTF-1 & CD10 & Other/Remarks & \\
\hline 50 & Kolin et al., 2019 [8] & $-1-$ & NA & + & NA & $\begin{array}{l}\text { P53 wild type } \\
\text { NapsinA - } \\
\text { WT1 - } \\
\text { Chromogranin - } \\
\text { Synaptophysin - } \\
\text { Thyroglobulin - }\end{array}$ & KRAS G12V \\
\hline 51 & Yamamoto et al., 2019 [29] & + very focal/- & + focal & + diffuse & + focal & $\begin{array}{l}\text { Calretinin focal + } \\
\text { Thyroglobulin - }\end{array}$ & NA \\
\hline $\begin{array}{l}52(53- \\
56 \mathrm{NA})\end{array}$ & $\begin{array}{l}\text { McCluggage et al., } \\
2020[4]\end{array}$ & $-1-$ & + focal & + focal & + luminal & $\begin{array}{l}\text { P53 wild type } \\
\text { WT1 - } \\
\text { Thyroglobulin - }\end{array}$ & KRAS G12D \\
\hline 57 & Dundr et al., 2020 [30] & $-1-$ & $+(30 \%)$ & $+(70 \%)$ & + focal & $\begin{array}{l}\text { P53 wild type } \\
\text { Calretinin - } \\
\text { WT1 - } \\
\text { HNF1b - } \\
\text { Inhibin - }\end{array}$ & $\begin{array}{l}\text { KRAS (c.34G > T, p.(G12C)) and PIK3CA } \\
\text { (c.1633G > A, p.(E545K)) } \\
\text { Likely pathogenic somatic MYCN mutation } \\
\text { (c.131C > T, p.(P44L) } \\
\text { Hereditary CHEK2 mutation. }\end{array}$ \\
\hline 58 & Seay et al., 2020 [31] & $-1-$ & + focal & + focal & + focal & $\begin{array}{l}\text { P16 patchy } \\
\text { WT1 - } \\
\text { Calretinin - } \\
\text { P53 wild type } \\
\text { MMRp } \\
\text { Preserved PTEN, } \\
\text { ARID1A }\end{array}$ & $\begin{array}{l}\text { Variants of unknown significance in the ATM } \\
\text { gene (c.4303A > C (p.Lys145Gln) } \\
\text { and PALB2 gene (c.693A > T (p.Lys231Asn) }\end{array}$ \\
\hline $59-127$ & $\begin{array}{l}\text { Pors et al., } 2020 \text { [10] } \\
44+25 \text { cases }\end{array}$ & Positive for at le & GATA3, & D10 (lumin & ND negative/foce & y for ER, or molecu & rmation (KRAS mutations) \\
\hline 128-131 & $\begin{array}{l}\text { Horn et al., } 2020 \text { [7] } \\
4 \text { cases }\end{array}$ & $-/-(4 / 4)$ & $-(2 / 2)$ & $+(4 / 4)$ & + luminal $(2 / 2)$ & $\begin{array}{l}\text { P16 patchy } \\
\text { P53 wild type } \\
\text { MMRp }\end{array}$ & $\begin{array}{l}\text { KRAS G12V (3/4) } \\
\text { KRAS G12D }(1 / 4)\end{array}$ \\
\hline
\end{tabular}


Table 3. Cont.

\begin{tabular}{|c|c|c|c|c|c|c|c|}
\hline & \multirow{2}{*}{ Case } & \multicolumn{5}{|c|}{ Immunohistochemistry (IHC) } & \multirow{2}{*}{ Molecular } \\
\hline & & ER/PR & GATA3 & TTF-1 & CD10 & Other/Remarks & \\
\hline $132-154$ & $\begin{array}{l}\text { Euscher et al., } 2020 \text { [6] } \\
23 \text { cases }\end{array}$ & $\begin{array}{l}-/-(11 / 23) \\
+/-(3 / 23) \\
+/+(1 / 23) \\
+/ \mathrm{ND}(2 / 23) \\
-/ \mathrm{ND}(4 / 23) \\
\mathrm{ND} / \mathrm{ND}(2 / 23) \\
\text { (ER }+10-40 \% \\
\text { focal to patchy) }\end{array}$ & \multicolumn{2}{|c|}{$\begin{array}{l}\text { GATA3+/TTF1+ }(10 / 23) \\
\text { GATA3+/TTF1-(4/23) } \\
\text { GATA3-/TTF1+ }(0 / 23) \\
\text { GATA3-/TTF1-(1/23) } \\
\text { GATA3 NA/TTF1+ }(1 / 23) \\
\text { GATA3+/TTF1 NA }(1 / 23) \\
\text { NA }(6 / 23)\end{array}$} & $\begin{array}{l}+ \text { luminal }(10 / 23) \\
\text { NA }(13 / 23)\end{array}$ & $\begin{array}{l}\text { Calretinin } \\
-(10 / 15) \\
+(5 / 15)\end{array}$ & $\begin{array}{l}\text { 13/17 KRAS } \\
-\quad \text { G12D }(7 / 13) \\
-\quad \text { G12V }(5 / 13) \\
-\quad \text { G12A }(1 / 13) \\
\text { Five cases with KRAS mutation also had } \\
\text { additional mutations including PIK3CA } \\
(3 / 5) ; \\
\text { PTEN }(2 / 5) \text { and CTNNB1 }(1 / 5) .\end{array}$ \\
\hline 155 & Our case & $-1-$ & + & - & - & $\begin{array}{l}\text { P53 wild type } \\
\text { PAX8 + } \\
\text { SATB2- } \\
\text { CK7 focal }+ \\
\text { CK20 focal }+ \\
\text { P16 patchy } \\
\text { Vimentin }+ \\
\text { MMRp }\end{array}$ & $\begin{array}{l}\text { KRAS G13N } \\
\text { Two probable pathogenic variants of PTEN } \\
(\text { c. } 388 \mathrm{C}>\mathrm{T} \text { and c. } 634+2 \mathrm{~T}>\mathrm{G}) .\end{array}$ \\
\hline
\end{tabular}

Italics: located in the myometrium. ND: not done; NA: not available; MMRp: mismatch repair proficient; +: positive; -: negative. 


\section{Discussion}

\subsection{Epidemiology}

Mesonephric-like adenocarcinomas are rare neoplasms with a reported incidence of $1 \%$ of all endometrial carcinomas [7,8]. In the literature, 115 uterine and 39 ovarian cases have been reported. Of these, 16 MLA had associated findings of Müllerian origin: adenomyosis [22,23], endometriosis [4,22,31], atypical hyperplasia of the endometrium (or EIN) [10], serous cystadenoma [4], mixed serous and mucinous cystadenoma [4], serous borderline tumor [3,4,30], borderline endometrioid adenofibroma [4], low-grade endometrioid endometrial carcinoma [28], low-grade serous ovarian carcinoma [3,4].

All age groups were affected, ranging from 26 to 91 years with a mean of 59 years and a median of 61 years.

\subsection{Pathogenesis}

The cell lineage of origin is still a matter of debate. With the morphology reminiscent of classic mesonephric carcinoma and overlapping immunohistochemical features, it could be a type of mesonephric carcinoma with divergent Müllerian features. Proteomic analysis of both MA and MLA was as good as identical [5]. On the other hand, uterine tumors tend to originate from the endometrium with secondary involvement of the myometrium and they are not associated with mesonephric remnants. Cases where the MLA is associated with other Müllerian lesions support the evidence of a Müllerian lesion that differentiated along the mesonephric lines. Yano et al., Dundr et al., McCluggage et al. and Chapel et al. could prove clonality between the Müllerian lesions (endometrioid endometrial carcinoma, serous borderline tumor and low-grade serous carcinoma of the ovary) since they share mutations in the KRAS and NRAS gene $[3,4,28]$.

\subsection{Morphology}

MLA shows considerable overlap with conventional mesonephric carcinomas. They are characterized by a variety of growth patterns, between tumors and within the same tumor composed of small tubules, ductal/glandular growth, papillary, solid growth, sex cord-like, trabecular, retiform, sieve-like, glomeruloid and spindle cell areas are described. Luminal eosinophilic colloid-like secretions are characteristic but not always present. Ductular/glandular and tubular patterns are most frequently described (Tables 2 and 3 [6]). The tumor cells may be flattened, cuboidal or columnar with usually scant eosinophilic cytoplasm. Focal cytoplasmic clearing is possible but rather rare [6,22]. There is mild to moderate cytological atypia. The nuclei can be oval to flattened, angulated with vesicular to optically clear chromatin, sometimes with nuclear groves or nuclear overlap. These nuclear features can be reminiscent of papillary thyroid carcinoma [6,22]. High-grade cytological atypia is normally not the predominant feature. Hobnail cells are a rarely reported feature $[6,30]$.

There should be no squamous, ciliated or mucinous differentiation (metaplasia) and no associated mesonephric remnants.

\subsection{Immunohistochemistry}

MLAs are usually positive for Paired box protein-8 (PAX8), GATA binding protein 3 (GATA3), thyroid transcription factor 1 (TTF1), CD10 with luminal staining, and are negative for estrogen receptor (ER) and progesterone receptor (PR). However, focal positivity of ER is described by Kolin et al. (2/4 cases with weak, patchy or heterogeneous staining [8]), Pors et al. (2/5 cases weak to moderate in 10-55\% [27]), Ando et al. (only a small number of cells $(<1 \%)$ in the tubular pattern expressed ER [23]), Euscher et al. (6/23 cases with ER ranging from $10 \%$ up to $40 \%$ [6]), Kenny et al. [18], Patel et al. (weak focal staining in $<5 \%$ [26]) and Yamamoto et al. (very focal [29]). Hence some positivity of ER does not preclude the diagnosis of MLA. PR was negative in all but two of these cases. So it can be concluded that PR is a more reliable negative marker for MLA. 
ER/PR negativity in endometrioid endometrial adenocarcinoma (EEC) is an independent risk factor for recurrence and death in FIGO grade I-II EEC [32]. However in these previous studies, no testing for GATA3 or TTF1 was performed, and so no definite conclusions can be drawn on how many of these ER/PR negative (low-grade) EEC constitute MLA.

Calretinin, CD10 and ER used to be the markers to diagnose MLA before recognition of the role of TTF1 and GATA3. Howitt et al. compared GATA3 expression in mesonephric/Wolffian remnant with other tumors of the female genital tract. They found that GATA3 has a sensitivity of $98 \%$ and a specificity of $98 \%$ to differentiate mesonephric lesions to endocervical and endometrial carcinomas [20], which was confirmed by the whole proteome analysis by Gibbard et al. [5]. Later Pors et al. compared the sensitivity and specificity of GATA3, TTF1, CD10 and calretinin in the diagnosis of MLAs and reported GATA3 to be the best overall marker, but staining can be weak to moderate in intensity and positive in only a minority of cells ( $<10 \%)$ [27]. This finding was confirmed by Euscher et al. [6]. TTF1 and GATA3 regularly show an inverse staining pattern $[6,26,27]$ and GATA3 is less expressed in more solid/spindled and sarcomatoid regions of the tumor $[9,20,27]$.

CD10 shows in most of the cases at least focal expression with staining of the luminal/apical surface and has a reported sensitivity of $73 \%$ and specificity of $83 \%$ [10]. Calretinin positivity can support the diagnosis but is frequently negative. Moreover, CD10 is more difficult to interpret due to the positivity of surrounding endometrial stroma and smooth muscle and calretinin can give a background nonspecific granular cytoplasmic staining.

The expression pattern of p53 is wild type, p16 shows patchy staining, WT1 is negative. MLA is typically MMR proficient, with normal expression of MLH1, MSH2, MSH6 and PMS2.

\subsection{Molecular Findings}

The majority of MLA harbor KRAS mutations, suggesting KRAS mutation is involved in MLA development. The KRAS mutation G12V and G12D are the most common, G12A and G12C are respectively 4 and 3 times reported. Concurrent ARID1A and PIK3CA mutations are relatively common and described in respectively nine and seven cases $[1,6,9,30]$. PTEN mutation, also frequent in EEC, was found as an additional mutation in three MLA cases $[6,9]$. In the case of $\mathrm{Na}$ et al., this was detected in metastatic tumor only, demonstrating that PTEN mutation is probably a relatively late event in the sequence of genetic alterations [9]. KRAS and ARID1A are common mutations in both MA and EEC, and so will not help in defining the mesonephric or Müllerian nature of MLA [2,33]. On the other hand, PIK3CA and PTEN mutations, which are common in EEC but have not been described in MA of the cervix are rather indicative of Müllerian origin with subsequent differentiation along mesonephric lines [33].

Copy number variation testing is increasingly being implemented. Copy number gain of $1 \mathrm{q}$ is most common $[1,3,8,9,33]$ and some of these have also $1 \mathrm{p}$ loss. The gain of chromosome 10 is found in metastatic disease and may be an indicator of aggressive biological behavior $[9,33]$.

Since these tumors have no aberrant p53 staining (no TP53 mutation), have no loss of mismatch repair protein expression and so far have no POLE exonuclease domain hotspot mutation (POLE), they belong to the molecular group of no specific molecular profile (NSPM), and are probably responsible for the proportion of poor survivals in this group.

\subsection{Prognosis}

MLAs have aggressive biological behavior with more than half of the published cases presented with advanced stage (FIGO $\geq$ II) at diagnosis. They are associated with a considerable risk of recurrent disease with a tendency to metastasize to the lungs [6-10]. Not only high stage disease but also stage I disease frequently metastasizes $[9,12,14,28,29,31]$. This was confirmed by Pors et al. who calculated that the stage at diagnosis was not 
significant for progression-free survival. They reported a 5-year overall survival of 71 to $72 \%$ for mesonephric adenocarcinomas of the uterine body and ovary [10]. Six characteristics were significantly associated with the development of metastasis, including large tumor size $(>4 \mathrm{~cm})$, ill-defined tumor border, advanced FIGO stages (III to IV), presence of coagulative tumor cell necrosis, high mitotic activity $(>10 / 10$ high-power fields), and presence of lymphovascular invasion. These high mitotic activities and lymphovascular invasion were found to be independent factors [9]. Compared with other endometrial adenocarcinomas, MLAs have better overall survival than malignant mixed Müllerian tumors and serous carcinoma has equal overall survival to endometrioid grade 3 and has worse overall survival than endometrioid grade 1-2 carcinomas [10]. Endometrial carcinomas have a tendency for lymphovascular metastasis to pelvic lymph nodes followed by retroperitoneal lymph nodes. Distant metastases in endometrial carcinoma are rare with a reported incidence of 3.1\% (all tumor types) [34]. Although the lungs are the most common site $(1.5 \%)$, only $1.1 \%$ of EEC do present with lung metastasis [34].

\subsection{Treatment}

All cases were treated with a total hysterectomy and bilateral salpingo-oophorectomy. Pelvic lymph node dissection was often added, potentially also with para-aortic lymph node dissection. Adjuvant chemotherapy, mainly carboplatin + paclitaxel, was given in high stage disease but also in one case of FIGO stage IA and two cases of FIGO stage IB $[9,23]$. Radiation therapy was given solo in early cases and in addition to chemotherapy in higher stage cases. Two reported cases were treated with hormone therapy $[6,28]$. One case was diagnosed as a low-grade EEC, the concurrent MLA component was only retrospectively recognized, and was treated with progesterone therapy; 6 years later only the MLA recurred [28]. The other case was also diagnosed as EEC, grade 1-2, the type of hormone therapy was not specified. This tumor recurred with distant metastasis to the liver after 17 months [6].

The optimal regimen and the efficacity of (neo) adjuvant radiation and or chemotherapy remains largely unknown. So far, no tumor-specific treatment options have been elucidated for MLA.

\subsection{Differential Diagnosis}

The diagnosis can be challenging due to the rarity and with the diverse histologic pattern, the tumor is also frequently under-recognized and misdiagnosed. There is some degree of morphologic overlap with EEC, clear cell carcinoma, serous carcinoma, as well as carcinosarcoma. Tubules with eosinophilic secretions are a diagnostic clue for MLA. Of course one must exclude cervical mesonephric adenocarcinoma with the involvement of the uterine corpus. This can be done macroscopically by determining where the tumor is predominantly located and needs to be correlated to imaging. Microscopically, mesonephric carcinomas of the cervix are frequently associated with mesonephric remnants/hyperplasia, while this should not be seen in MLA. Most cases of MLA of the uterine body originate in the endometrium and cases described as MA of the uterine corpus originate more in the lateral walls in the myometrium, where mesonephric remnants are to be expected. In this review tumors that originate in the myometrium but where no mesonephric remnants near the tumor were found are also included for completeness of mesonephric lesions in the uterine corpus, but these could also be true MA instead of MLA (see italics in Tables 1 and 2). Both MA and MLA have negative ER and PR staining and express GATA3. Calretinin and CD10 may also be positive in both MLA and MA. MLAs have in comparison to MA more frequently TTF1 positive staining [27]. For further differentiation, additional molecular testing can be performed. MLA and MA share KRAS mutations, but when PTEN and PIK3CA mutations are found the diagnosis of MLA is made above a MA.

The main differential diagnosis is EEC with the tubular and glandular growth pattern. MLA has tubules and glands as the most common feature but is characterized by com- 
mon heterogeneity of architectural patterns. Cytological features of nuclei with vesicular chromatin and nuclear grooves seen in MLA are not characteristic of endometrial carcinoma, while EECs are usually composed of cells that are columnar with pseudostratified nuclei. When squamous, ciliated or mucinous differentiation is seen, MLA can be excluded. Endometrial hyperplasia or endometrioid intraepithelial neoplasia are precursor lesions for EEC, however there are reports where these are found with MLA (including the case presented in this manuscript) [10], but lack of these favors MLA. Immunohistochemically, MLA is characterized by GATA-3 and/or TTF1 expression, which is rare in EEC [20,27]. EECs are normally positive for estrogen and progesterone receptors, which are nearly always absent in MLA, with negative PR as the most reliable marker. So when diffuse and strong ER and PR are found, a diagnosis of MLA is very unlikely. GATA3 expression can be seen in a minority of endometrial carcinomas: 6\% reported by Pors et al., including endometrioid adenocarcinomas, but these cases were always TTF1 negative [10,20]. Terzik et al. reported GATA3 positivity in endometrial premalignant and malignant proliferations with an incidence of $8 \%$ (5 of 64 cases): one with atypical hyperplasia, one high-grade endometrioid adenocarcinoma, two serous carcinomas and one carcinosarcoma. Additionally, GATA3 expression in EECs is not diffuse but focal to patchy with weak to strong staining [35]. For TTF1 Pors et al. reported only 1.0\% (6 of 585 cases) of endometrial neoplasms with TTF1 expression, including three endometrioid carcinomas, one serous carcinoma, one clear cell carcinoma and one carcinosarcoma [27]. Other earlier studies reported TTF1 expression ranging from 2\% up to 19\% in EEC and 9-23\% in serous carcinomas and 7\% clear cell carcinoma [36,37]. These EECs with TTF1 expression are reported to have a worse prognosis [36]. It could be possible that some of these may actually represent MLA since the cases of Ervine et al. [36] were all ER-negative and there was no additional immunohistochemical staining for GATA3, PR, CD10 or calretinin reported.

When the papillary architectural pattern in association with high-grade nuclear atypia is observed, serous endometrial carcinoma should be considered. Serous carcinoma is characterized by p53 mutation and p16 block-staining, which is never seen in MLA. ER and PR are not helpful in differentiating because both MLA and serous carcinomas share negative hormone receptor expression.

Clear cell carcinomas can also have a combination of architectural patterns, with variable cytological atypia and a low mitotic index. Hobnail cells and cytoplasmic clearing are seldom seen in MLA but can raise the possibility of clear cell carcinoma. Immunohistochemically, clear cell carcinomas are typically positive for HNF-1B, and often for napsin $A$ and/or Alpha methyacyl CoA racemase (AMACR). Napsin A and HNF-1b are mostly negative in MLA, but can be positive. Clear cell carcinomas also show negative ER/PR staining, but can have abnormal p53 and can be mismatch repair deficient, in contrast to MLA that always have wild type p53 staining and the reported cases are mismatch repair proficient. GATA3 and TTF1 are usually negative in clear cell carcinomas.

With solid areas and spindled cell and sarcomatoid features, MLA can be confused with carcinosarcoma. Lack of heterologous differentiation, as well as wild type p53 staining, suggests the tumor is less likely a carcinosarcoma. Moreover, p53 wild-type carcinosarcomas often demonstrate microsatellite instability (MSI) (rather representing undifferentiated or dedifferentiated carcinomas), and do not appear to harbor KRAS mutations [38].

In a metastatic setting, like in our case, with metastasis to the lungs, the positive TTF1 staining and negative hormone receptors can be confusing with primary lung adenocarcinoma. In patients with a history of gynecological malignancy one should always perform PAX8 staining. The morphology of pseudoendometrioid glands and small glands with eosinophilic secretions give a clue for MLA. Additional GATA3 staining, CD10 and calretinin staining can further help to support the diagnosis of MLA.

\section{Conclusions}

Features that should make the pathologist think about the possibility of MLA are the presence of a combination of architectural patterns, with most frequently ductu- 
lar/glandular and tubular growth pattern, in a tumor without squamous of mucinous differentiation. A diagnostic clue is the presence of intraluminal dense eosinophilic secretions. When such morphology is observed, additional immunohistochemical staining can be performed. We suggest using PAX8, GATA3, TTF1, ER and PR as first-line markers, as proposed by Pors et al. 2018 [27]. When positive, CD10 and calretinin might be helpful additional markers. The tumor should be MMR proficient. Molecular analysis with the finding of KRAS and possibly PIK3CA, ARID1A or PTEN mutation can support the diagnosis.

Further investigation is needed for endometrial tumors that have a loss of hormone receptors and positive staining for GATA3 and/or TTF1, with p53 wild type pattern and MMR proficient. Are all these tumors, with histology that fits MLA as well as other subtypes of endometrial tumors, by definition MLA?

Mesonephric-like adenocarcinomas are considered high-grade carcinomas, even though they have a misleadingly low-grade morphology. The tumors have a high risk of recurrence and a high tendency for lung metastasis. Further research on the pathogenesis should help better understand this specific subset of endometrial cancer. As of today, tumor-specific treatment options are limited and the best therapeutic strategy is yet to be determined.

Author Contributions: Conceptualization, E.D., J.V.D. and K.V.d.V.; methodology, E.D. and K.V.d.V.; formal analysis, E.D. and K.V.d.V.; investigation, E.D., J.V.D. and K.V.d.V.; resources, E.D.; data curation, E.D. and K.V.d.V.; writing—original draft preparation, E.D.; writing—review and editing, E.D., J.V.D. and K.V.d.V.; visualization, E.D., J.V.D. and K.V.d.V.; supervision, J.V.D. and K.V.d.V.; project administration, K.V.d.V.; funding acquisition, K.V.d.V. All authors have read and agreed to the published version of the manuscript.

Funding: This research received no external funding.

Institutional Review Board Statement: Ethical review and approval were waived for this study. It is a review paper using exsisting literature.

Informed Consent Statement: Informed consent was obtained from the patient involved in the study to described the case anonymously.

Data Availability Statement: No new data were created or analyzed in this study. Data sharing is not applicable to this article.

Conflicts of Interest: The authors declare no conflict of interest.

\section{References}

1. Mirkovic, J.; McFarland, M.; Garcia, E.; Sholl, L.M.; Lindeman, N.; MacConaill, L.; Dong, F.; Hirsch, M.; Nucci, M.R.; Quick, C.M.; et al. Targeted genomic profiling reveals recurrent KRAS mutations in mesonephric-like adenocarcinomas of the female genital tract. Am. J. Surg. Pathol. 2018, 42, 227-233. [CrossRef] [PubMed]

2. Kandoth, C.; Schultz, N.; Cherniack, A.D.; Akbani, R.; Liu, Y.; Shen, H.; Robertson, A.G.; Pashtan, I.; Shen, R.; Benz, C.C.; et al. Integrated genomic characterization of endometrial carcinoma. Nature 2013, 497, 67-73. [PubMed]

3. Chapel, D.B.; Joseph, N.M.; Krausz, T.; Lastra, R.R. An ovarian adenocarcinoma with combined low-grade serous and mesonephric morphologies suggests a müllerian origin for some mesonephric carcinomas. Int. J. Gynecol. Pathol. Off. J. Int. Soc. Gynecol. Pathol. 2018, 37, 448-459. [CrossRef] [PubMed]

4. McCluggage, W.G.; Vosmikova, H.; Laco, J. Ovarian combined low-grade serous and mesonephric-like Adenocarcinoma: Further evidence for a mullerian origin of mesonephric-like adenocarcinoma. Int. J. Gynecol. Pathol. Off. J. Int. Soc. Gynecol. Pathol. 2020, 39, 84-92. [CrossRef] [PubMed]

5. Gibbard, E.; Cochrane, D.R.; Pors, J.; Negri, G.L.; Colborne, S.; Cheng, A.S.; Chow, C.; Farnell, D.; Tessier-Cloutier, B.; McAlpine, J.N.; et al. Whole-proteome analysis of mesonephric-derived cancers describes new potential biomarkers. Human Pathol. 2020, 108, 1-11. [CrossRef] [PubMed]

6. Euscher, E.D.; Bassett, R.; Duose, D.Y.; Lan, C.; Wistuba, I.; Ramondetta, L.; Ramalingam, P.; Malpica, A. Mesonephric-like carcinoma of the endometrium: A subset of endometrial carcinoma with an aggressive behavior. Am. J. Surg. Pathol. 2020, 44, 429443. [CrossRef] [PubMed]

7. Horn, L.C.; Höhn, A.K.; Krücken, I.; Stiller, M.; Obeck, U.; Brambs, C.E. Mesonephric-like adenocarcinomas of the uterine corpus: Report of a case series and review of the literature indicating poor prognosis for this subtype of endometrial adenocarcinoma. J. Cancer Res. Clin. Oncol. 2020, 146, 971-983. [CrossRef] [PubMed] 
8. Kolin, D.L.; Costigan, D.C.; Dong, F.; Nucci, M.R.; Howitt, B.E. A combined morphologic and molecular approach to retrospectively identify KRAS-mutated mesonephric-like adenocarcinomas of the endometrium. Am. J. Surg. Pathol. 2019, 43, 389-398. [CrossRef] [PubMed]

9. Na, K.; Kim, H.S. Clinicopathologic and molecular characteristics of mesonephric adenocarcinoma arising from the uterine body. Am. J. Surg. Pathol. 2019, 43, 12-25. [CrossRef] [PubMed]

10. Pors, J.; Segura, S.; Chiu, D.S.; Almadani, N.; Ren, H.; Fix, D.J.; Howitt, B.E.; Kolin, D.; McCluggage, W.G.; Mirkovic, J.; et al. Clinicopathologic characteristics of mesonephric adenocarcinomas and mesonephric-like adenocarcinomas in the gynecologic tract: A multi-institutional study. Am. J. Surg. Pathol. 2020. [CrossRef]

11. WHO. Classification of Tumours Editorial Board. WHO Classification of Tumours: Female Genital Tumours, 5th ed.; WHO: Geneva, Switzerland, 2020; Volume 4.

12. Yamamoto, Y.; Akagi, A.; Izumi, K.; Kishi, T. Carcinosarcoma of the uterine body of mesonephric origin. Pathol. Int. 1995, 45, 303-309. [CrossRef]

13. Ordi, J.; Nogales, F.F.; Palacin, A.; Márquez, M.; Pahisa, J.; Vanrell, J.A.; Cardesa, A. Mesonephric adenocarcinoma of the uterine corpus: CD10 expression as evidence of mesonephric differentiation. Am. J. Surg. Pathol. 2001, 25, 1540-1545. [CrossRef]

14. Montagut, C.; Mármol, M.; Rey, V.; Ordi, J.; Pahissa, J.; Rovirosa, A.; Gascón, P.; Mellado, B. Activity of chemotherapy with carboplatin plus paclitaxel in a recurrent mesonephric adenocarcinoma of the uterine corpus. Gynecol. Oncol. 2003, 90, 458-461. [CrossRef]

15. Bagué, S.; Rodríguez, I.M.; Prat, J. Malignant mesonephric tumors of the female genital tract: A clinicopathologic study of 9 cases. Am. J. Surg. Pathol. 2004, 28, 601-607. [CrossRef] [PubMed]

16. Marquette, A.; Moerman, P.; Vergote, I.; Amant, F. Second case of uterine mesonephric adenocarcinoma. Int. J. Gynecol. Pathol. Off. J. Int. Soc. Gynecol. Pathol. 2006, 16, 1450-1454.

17. Wani, Y.; Notohara, K.; Tsukayama, C. Mesonephric adenocarcinoma of the uterine corpus: A case report and review of the literature. Int. J. Gynecol. Pathol. Off. J. Int. Soc. Gynecol. Pathol. 2008, 27, 346-352. [CrossRef] [PubMed]

18. Kenny, S.L.; McBride, H.A.; Jamison, J.; McCluggage, W.G. Mesonephric adenocarcinomas of the uterine cervix and corpus: HPV-negative neoplasms that are commonly PAX8, CA125, and HMGA2 positive and that may be immunoreactive with TTF1 and hepatocyte nuclear factor 1- $\beta$. Am. J. Surg. Pathol. 2012, 36, 799-807. [CrossRef] [PubMed]

19. Wu, H.; Zhang, L.; Cao, W.; Hu, Y.; Liu, Y. Mesonephric adenocarcinoma of the uterine corpus. Int. J. Clin. Exp. Pathol. 2014, 7, 7012-7019. [PubMed]

20. Howitt, B.E.; Emori, M.M.; Drapkin, R.; Gaspar, C.; Barletta, J.A.; Nucci, M.R.; McCluggage, W.G.; Oliva, E.; Hirsch, M.S. GATA3 is a sensitive and specific marker of benign and malignant mesonephric lesions in the lower female genital tract. Am. J. Surg. Pathol. 2015, 39, 1411-1419. [CrossRef] [PubMed]

21. Kim, S.S.; Nam, J.H.; Kim, G.; Choi, Y.D.; Choi, C.; Park, C.S. Mesonephric adenocarcinoma of the uterine corpus: A case report and diagnostic pitfall. Int. J. Surg. Pathol. 2016, 24, 153-158. [CrossRef] [PubMed]

22. McFarland, M.; Quick, C.M.; McCluggage, W.G. Hormone receptor-negative, thyroid transcription factor 1-positive uterine and ovarian adenocarcinomas: Report of a series of mesonephric-like adenocarcinomas. Histopathology 2016, 68, 1013-1120. [CrossRef] [PubMed]

23. Ando, H.; Watanabe, Y.; Ogawa, M.; Tamura, H.; Deguchi, T.; Ikeda, K.; Fujitani, M.; Shioji, M.; Tsujie, T.; Doi, R.; et al. Mesonephric adenocarcinoma of the uterine corpus with intracystic growth completely confined to the myometrium: A case report and literature review. Diag. Pathol. 2017, 12, 63. [CrossRef] [PubMed]

24. Kim, G.; Veran-Taguibao, S.; Taguibao, R. A rare case of primary uterine mesonephric adenocarcinoma. Am. J. Clin. Pathol. 2018, 149 (Suppl. 1), S113-S114. [CrossRef]

25. Zhang, L.; Cai, Z.; Ambelil, M.; Conyers, J.; Zhu, H. Mesonephric adenocarcinoma of the uterine corpus: Report of 2 cases and review of the literature. Int. J. Gynecol. Pathol. Off. J. Int. Soc. Gynecol. Pathol. 2019, 38, 224-229. [CrossRef] [PubMed]

26. Patel, V.; Kipp, B.; Schoolmeester, J.K. Corded and hyalinized mesonephric-like adenocarcinoma of the uterine corpus: Report of a case mimicking endometrioid carcinoma. Human Pathol. 2019, 86, 243248. [CrossRef] [PubMed]

27. Pors, J.; Cheng, A.; Leo, J.M.; Kinloch, M.A.; Gilks, B.; Hoang, L. A comparison of GATA3, TTF1, CD10, and calretinin in identifying mesonephric and mesonephric-like carcinomas of the gynecologic tract. Am. J. Surg. Pathol. 2018, 42, 1596-1606. [CrossRef]

28. Yano, M.; Shintani, D.; Katoh, T.; Hamada, M.; Ito, K.; Kozawa, E.; Hasegawa, K.; Yasuda, M. Coexistence of endometrial mesonephric-like adenocarcinoma and endometrioid carcinoma suggests a Müllerian duct lineage: A case report. Diag. Pathol. 2019, 14, 54. [CrossRef]

29. Yamamoto, S.; Sakai, Y. Pulmonary metastasis of mesonephric-like adenocarcinoma arising from the uterine body: A striking mimic of follicular thyroid carcinoma. Histopathology 2019, 74, 651-653. [CrossRef] [PubMed]

30. Dundr, P.; Gregová, M.; Němejcová, K.; Bártů, M.; Hájková, N.; jan Hojný; Stružinská, I.; Fischerová, D. Ovarian mesonephric-like adenocarcinoma arising in serous borderline tumor: A case report with complex morphological and molecular analysis. Diag. Pathol. 2020, 15, 91. [CrossRef] [PubMed]

31. Seay, K.; Akanbi, T.; Bustamante, B.; Chaudhary, S.; Goldberg, G.L. Mesonephric-like adenocarcinoma of the ovary with co-existent endometriosis: A case report and review of the literature. Gynecol. Oncol. Rep. 2020, 34, 100657. [CrossRef] [PubMed] 
32. Guan, J.; Xie, L.; Luo, X.; Yang, B.; Zhang, H.; Zhu, Q.; Chen, X. The prognostic significance of estrogen and progesterone receptors in grade I and II endometrioid endometrial adenocarcinoma: Hormone receptors in risk stratification. J. Gynecol. Oncol. 2019, 30, e13. [CrossRef] [PubMed]

33. Mirkovic, J.; Lindeman, N.; MacConaill, L.; Hirsch, M.; Cin, P.D.; Gorman, M.; Barletta, J.A.; Nucci, M.R.; McCluggage, W.G.; Howitt, B.E.; et al. Targeted genomic profiling reveals recurrent KRAS mutations and gain of chromosome 1q in mesonephric carcinomas of the female genital tract. Modern pathology. Off. J. US Canad. Acad. Pathol. Inc. 2015, 28, 1504-1514. [CrossRef] [PubMed]

34. Mao, W.; Wei, S.; Yang, H.; Yu, Q.; Xu, M.; Guo, J.; Gao, L. Clinicopathological study of organ metastasis in endometrial cancer. Future Oncol. 2020, 16, 525-540. [CrossRef] [PubMed]

35. Terzic, T.; Mills, A.M.; Zadeh, S.; Atkins, K.A.; Hanley, K.Z. GATA3 expression in common gynecologic carcinomas: A potential pitfall. Int. J. Gynecol. Pathol. Off. J. Int. Soc. Gynecol. Pathol. 2019, 38, 485-492. [CrossRef] [PubMed]

36. Ervine, A.; Leung, S.; Gilks, C.B.; McCluggage, W.G. Thyroid transcription factor-1 (TTF-1) immunoreactivity is an adverse prognostic factor in endometrioid adenocarcinoma of the uterine corpus. Histopathology 2014, 64, 840-846. [CrossRef] [PubMed]

37. Siami, K.; McCluggage, W.G.; Ordonez, N.G.; Euscher, E.D.; Malpica, A.; Sneige, N.; Silva, E.G.; Deavers, M.T. Thyroid transcription factor-1 expression in endometrial and endocervical adenocarcinomas. Am. J. Surg. Pathol. 2007, 31, 1759-1763. [CrossRef] [PubMed]

38. Cherniack, A.D.; Shen, H.; Walter, V.; Stewart, C.; Murray, B.A.; Bowlby, R.; Hu, X.; Ling, S.; Soslow, R.A.; Broaddus, R.R.; et al. Integrated molecular characterization of uterine carcinosarcoma. Cancer Cell. 2017, 31, 411-423. [CrossRef] 